\title{
BMJ Open RAndomised controlled trial to imProve depressIon and the quality of life of people with Dementia using cognitive bias modification: RAPID study protocol
}

\author{
Osvaldo P Almeida, ${ }^{1,2,3}$ Colin MacLeod, ${ }^{4}$ Leon Flicker, ${ }^{1,5,6}$ Andrew Ford, ${ }^{1,2,3}$ \\ Ben Grafton, ${ }^{4}$ Christopher Etherton-Beer ${ }^{1,5,6}$
}

To cite: Almeida OP, MacLeod C, Flicker L, et al. RAndomised controlled trial to imProve depresslon and the quality of life of people with Dementia using cognitive bias modification: RAPID study protocol. BMJ Open 2014;4:e005623. doi:10.1136/bmjopen-2014005623

- Prepublication history for this paper is available online. To view these files please visit the journal online (http://dx.doi.org/10.1136/ bmjopen-2014-005623).

Received 5 May 2014 Revised 20 June 2014 Accepted 4 July 2014

CrossMark

For numbered affiliations see end of article.

Correspondence to

Professor Osvaldo P Almeida; osvaldo.almeida@uwa.edu.au

\section{ABSTRACT}

Introduction: Depressive symptoms are common and undermine the quality of life of people with Alzheimer's disease (AD). Cholinesterase inhibitors and antidepressants have all but no effect on the mood of patients, and their use increases adverse events. Cognitive bias modification (CBM) targets attentional and interpretative biases associated with anxiety, dysphoria and depression and may be useful to treat depression in $A D(D A D)$. This trial aims to determine the effect of CBM on depression scores and the quality of life of people with DAD.

Methods and analysis: Randomised, double-blind, parallel, controlled trial of CBM (1:1 allocation ratio). Participants will be 80 adults with probable AD living in the Western Australian community who score 8 or more on the Cornell Scale for Depression in Dementia (CSDD). They will have mild to moderate dementia (Mini-Mental State Examination-MMSE score $\geq 15$ ) and will be free of severe sensory impairment or suicidal intent. The intervention will consist of 10 40 min sessions of CBM delivered over 2 weeks using a high-resolution monitor using a local computer station at the Western Australian Centre for Health and Ageing. The primary outcomes of interest are the 2week change, from baseline, in the severity of CSDD scores and the Quality of Life AD (QoL-AD) scores. Secondary outcomes include changes in the CSDD, QoL-AD after 12 weeks, and changes in MMSE scores, negative attentional and interpretative bias and the proportion of participants with CSDD $<8$ after 2 and 12 weeks.

Ethics and dissemination: The study will comply with the principles of the Declaration of Helsinki and participants will provide written informed consent. The Ethics Committee of the Royal Perth Hospital will approve and oversee the study (REG14-036). The results of this trial will provide level 2 evidence of efficacy for CBM as a treatment of DAD.

Trial registration number: Australian and New Zealand Clinical Trials Registry number ACTRN12614000420640, date registered 06/04/2014.

\section{Strengths and limitations of this study}

Strong empirical evidence supports the testing of cognitive bias modification as a treatment for depression in Alzheimer's disease.

- Cognitive bias modification interventions are not associated with clinically significant adverse events.

- The pragmatic definition of depression in this trial will be based on the use of a validated cutpoint on the Cornell Scale for Depression in Dementia rather than diagnostic criteria.

- The trial will be limited to people with depression in Alzheimer's disease of mild to moderate severity.

- The intervention will be limited to 2 weeks.

\section{INTRODUCTION}

Dementia is a leading cause of disability, affecting as many as $5 \%$ of the population over the age of 65 and $40 \%$ of those older than 80 years. ${ }^{12}$ As the World's population continues to age, the number of people living with dementia is expected to increase exponentially over the next 40 years, ${ }^{3}$ and this will lead to growing demands on residential care and health services. ${ }^{4}$ In addition, the characteristic cognitive decline that affects people with dementia is commonly accompanied by other mental health changes involving perception, reasoning, behaviour and mood. ${ }^{5}$ Most relevant to the current proposal is the fact that about 25\% of people with Alzheimer's disease (AD), which is the most frequent cause of dementia in Western societies, show evidence of clinically significant depressive symptoms at assessment independent of the level of severity of the disease. ${ }^{5}$ Currently available data also 
show that symptoms of anxiety and depression tend to co-occur in $\mathrm{AD}$ and may affect as many as 1 in every 2 people during the course of the illness. ${ }^{7}$ Worryingly, the presence of mood disturbances in $\mathrm{AD}$ increases disability and burden of care, ${ }^{8}$ impairs quality of life ${ }^{9}$ and has been associated with accelerated cognitive decline in longitudinal studies. ${ }^{10}$ As currently available treatments for $\mathrm{AD}$ do not affect disease progression, ${ }^{11}$ it is important that we develop interventions that improve the quality of life of these patients.

\section{Treatments for AD do not ameliorate psychological comorbidity}

Medications commonly used to treat people with $\mathrm{AD}$ have no obvious effect on mood. The cholinesterase inhibitors donepezil, galantamine and rivastigmine improve the cognitive scores of patients with $\mathrm{AD}$ compared with placebo, ${ }^{12}$ but have no consistent impact on comorbid psychological and behavioural symptoms, including depression. ${ }^{13}$ Similarly, data from randomised controlled trials suggest that memantine does not possess antidepressant properties. ${ }^{14}$

\section{Treatments for depression associated with AD do not work and cause more side effects}

A large multicentre trial of the antidepressants sertraline and mirtazapine for the treatment of depression associated with dementia failed to show any benefit of treatment. ${ }^{15}$ The investigators randomly assigned 326 people with $\mathrm{AD}$ and depression to treatment with sertraline ( $\mathrm{n}=107$, up to $150 \mathrm{mg}$ daily), mirtazapine ( $\mathrm{n}=108$, up to $45 \mathrm{mg}$ daily) or placebo $(\mathrm{n}=111)$. Thirty-nine weeks of follow-up data were available. Treatment with antidepressants did not reduce depression scores relative to placebo after 13 or 39 weeks of treatment, but sertraline and mirtazapine were associated with greater frequency of adverse reactions than placebo $(43 \%, 41 \%$ and $26 \%$, respectively). Moreover, a systematic review of randomised placebo-controlled trials for the treatment of depression in dementia showed that the efficacy of antidepressants is equivalent to that of placebo over a period of 6-12 weeks. ${ }^{16}$ These negative results are alarming, as depression is commonly associated with $\mathrm{AD}$, hinders quality of life and is a significant source of stress for carers. ${ }^{8}$ Novel and effective approaches to manage these patients are needed.

\section{The contribution of cognitive bias to depression}

Various psychological interventions have been tested for the treatment of depression until now, with the most robust empirical evidence for efficacy arising from trials of cognitive-behavioural therapy (CBT). ${ }^{17} \mathrm{CBT}$ uses a systematic approach to cognitive restructuring, change of biased negative beliefs and behavioural activation to mitigate the intensity and presence of depression. ${ }^{18}$ Meta-analysis of pooled data of published trials indicates that CBT may also contribute to decreasing the risk of relapse over 12-24 months, suggesting that its benefits might extend beyond the acute phase of treatment. ${ }^{19}$ The theoretical framework underpinning CBT recognises that depressed mood becomes apparent when a set of biased beliefs and thinking processes predominate, commonly involving preferential attention to negative aspects of experience and an inflated tendency to impose negative interpretations to ambiguous events. Such biases in the processing of information initiate the cycle of negative thinking and behavioural changes that are characteristic of depression. CBT challenges negative thoughts about the self, others and the future with the aim of modifying biased ways of thinking and, consequently, leading to improved mood and behaviour. Effective shifts of dysfunctional biased beliefs have been associated with robust response to treatment and decreased risk of relapse of symptoms in cognitively intact people able to engage with the demands and requirements of the therapy. ${ }^{20}$ However, a substantial gap persists in treatment options for depressed adults with cognitive impairment.

Cognitive models of depression ascribe the development of symptoms to systematic biases in low-level mechanisms not readily accessible to conscious introspection that operate before thoughts are formed in ways that shape their nature. ${ }^{21}$ Selective biases in attention and interpretation that favour the processing of emotionally negative information are believed to represent the psychological basis of disordered mood, and emerging empirical and clinical findings support this hypothesis. ${ }^{22}$ People with depression have more difficulty keeping their attention away from negative stimuli than non-depressed people. For example, adults with depression show a series of faces on a computer screen selectively direct attention to sad faces, but show no such bias when presented with angry or happy faces. ${ }^{23}$ In addition, when faced with ambiguity, people with depressed mood favour negative interpretations of stimuli. For example, when presented with the ambiguous cue word 'GROWTH' (which can be interpreted negatively to mean tumour or non-negatively to mean increased size or wealth), people with low mood are significantly faster to then complete fragments of semantically related words associated with the negative rather than the nonnegative meanings of the ambiguous cue: C_NC_ER and GR_AT_R (cancer and greater). ${ }^{24}$ These findings invite the question: are these biases amenable to change, and could they be a target for the treatment of dysphoria and depression in people with dementia?

\section{What is cognitive bias modification?}

MacLeod and colleagues introduced the most widely used approach worldwide to modify attentional bias. ${ }^{25}$ The procedure exposes participants to pairs of words or images on a computer screen for $500 \mathrm{~ms}$, with each pair including one emotionally negative and one neutral item. Immediately after the words/images disappear, a single small visual probe is presented in the same spatial position where one of the original stimuli had been 
displayed. Participants are required to indicate, as quickly as possible, the orientation of this probe (horizontal or vertical), and their speed to accurately do so is recorded over dozens of trials. People who display an attentional bias to the more negative information are significantly faster to make this discrimination judgment for probes that appear in the same area as the negative stimuli compared with probes in the region of the neutral stimuli. Cognitive bias modification for attention (CBM-A) delivers hundreds of trials in which all probes are presented where the neutral rather than the negative stimuli had just appeared (promoting attentional avoidance of negative information). A control condition presents probes with equal frequency to each of these two areas. In CBM to reduce negative interpretative bias (CBM-I), participants are exposed to ambiguous information, followed by a word fragment that must be completed in a semantically consistent manner. CBM-I delivers hundreds of trials in which fragments yield only words consistent with non-negative interpretations of the ambiguity (discouraging negative interpretation). Thus, the ambiguous text "When you chat to people at a party they are soon chuckling, because you are so," will be followed by the fragment W_T_Y (yielding WITTY as opposed to 'silly'). A control condition employs fragments that equally often yield words consistent with negative or non-negative interpretation of the ambiguity. Single sessions of CBM can reduce negative attentional and interpretive bias, although multiple sessions may be associated with more lasting change. ${ }^{25}$

\section{CBM improves mood: previous trial evidence}

Recent trial data show that CBM-A attenuates anxiety reactions to stressful life events, ${ }^{26}$ reduces recurrent negative thought intrusions in chronic worriers, ${ }^{27}$ decreases avoidant behaviours ${ }^{28}$ and mitigates the intensity of depressive and dysphoric symptoms over a 2-week period. ${ }^{29}$ CBM is also effective at mitigating depressive symptoms in people with major depressive disorder. Williams et $a l^{30}$ randomised adults to CBM $(\mathrm{n}=38)$ or to a wait-list $(\mathrm{n}=31)$. CBM sessions were delivered daily over the internet for 1 week, and were associated with greater reduction in the severity of depressive symptoms.

\section{Rationale for the proposed trial}

Depression commonly affects people with AD during the course of their illness. Data from randomised controlled trials show that treatment with cholinesterase inhibitors, memantine and antidepressants is ineffective at reducing the severity of depressive symptoms, while the use of traditional forms of psychotherapy is hindered by the cognitive deficits that characterise $\mathrm{AD}$. As the pronounced impairment of explicit declarative learning (ie, episodic memory) associated with $\mathrm{AD}$ does not compromise the implicit learning that occurs when patients acquire cue-outcome associations, ${ }^{31}$ CBM may be a particularly suitable for use in this population.

\section{Objectives}

This trial aims to determine the effect of CBM on depressive symptoms and the quality of life of adults with depression in $\mathrm{AD}(\mathrm{DAD})$ after 2 weeks of treatment. We hypothesise that participants treated with active compared with control CBM will experience greater improvement of depressive symptoms and quality of life scores after 2 weeks of treatment. We also anticipate that the improvements in depression and quality of life scores will be maintained for 12 weeks and that these changes will be associated with a relative reduction in negative attentional and interpretative biases.

\section{METHODS}

\section{Study setting}

This study has been designed as a single centre trial based at the Western Australian Centre for Health \& Ageing at the Royal Perth Hospital in Australia. Participants will be community-dwelling older adults with $\mathrm{AD}$ in contact with the metropolitan health services.

\section{Trial design}

The RAndomised controlled trial to imProve depressIon and the quality of life of people with Dementia (RAPID) is a parallel, double-blind, controlled randomised trial of CBM with a 1:1 allocation ratio.

\section{Eligibility criteria}

We will recruit 80 people with depression in mild to moderate severity $\mathrm{AD}$ according to the following inclusion criteria:

1. Diagnosis of probable $\mathrm{AD}$ according to NINCDS-ADRDA criteria, ${ }^{32}$ which is largely consistent with DSM-5 diagnosis of major neurocognitive disorder due to probable $\mathrm{AD}$;

2. Mini-Mental State Examination (MMSE) score $\geq 15^{33}$;

3. Cornell Scale for Depression in Dementia (CSDD) $\geq 8^{34}$;

4. Fluent in written and spoken English (preferred language for at least 10 years).

We will exclude from participation people who have:

1. One or more diseases likely to compromise ongoing participation in the trial (eg, severe visual impairment),

2. A weekly alcohol consumption greater than 28 standard drinks ( $>4$ drinks per day) or 6 or more standard drinks on any 1 day of the week,

3. Active suicidal intent,

4. No health practitioner who is able to provide ongoing clinical care,

5. Changed antidepressants during the preceding 4 weeks,

6. Not been able or willing to provide informed consent to participate.

Participants will be recruited from metropolitan Memory Clinics and through advertisement via local health services, carer groups and the media. 


\section{Interventions}

Participants randomly assigned to the active and control CBM interventions will be exposed to the same study procedures and daily activities. They will be asked to attend the Western Australian Centre for Health and Ageing, Royal Perth Hospital, on a daily basis for a total of $10 \mathrm{CBM}$ sessions (2 weeks-excluding weekends). Each session will be delivered on a $24^{\prime \prime}$ high-resolution screen using a local PG station, and will last approximately 30 min: 15 min each for CBM-A and for CBM-I.

In the CBM-A session, participants will be shown pairs of emotionally discrepant photos for $500 \mathrm{~ms}$ (sad or neutral/happy faces). Each pair will then be replaced by a small probe (square or circle) appearing in the screen position previously occupied by one of the photos. Participants will be instructed to use a response box to indicate the shape of the small probe (by pressing the circle or square buttons of the response box). The probe will then disappear and will be replaced, after $1 \mathrm{~s}$, by another pair that of photos to initiate the next trial. The time to discriminate probe identity will be recorded automatically. In the active CBM condition, designed to reduce attention to negative information, probes will always appear in the position of neutral/happy faces. In the control condition, probes will appear $50 \%$ of the time in the position of neutral/happy faces and $50 \%$ of the time in the position of the sad faces.

In the interpretative CBM sessions (CBM-I), single ambiguous cue-words that permit a negative and benign interpretation (eg, HIT) will first appear in the top half of the screen for $1 \mathrm{~s}$. Then two words will appear on the bottom half of the screen: one on the left and the other on the right hand side. This word-pair will consist of one target word that is semantically related either to the negative or benign meaning of the ambiguous cue-word (eg, SUCCESS or PUNISH), and one foil word that is unrelated to either meaning of the ambiguous cue-word (eg, CLOUD). Participants will be asked to identify which is the target word and use the response box to indicate whether the semantically-related word appeared on the left or right-hand side of the screen (by pressing the left or the right hand side button of the response box, respectively). The time required to accurately identify the target word will be recorded automatically. In the active CBM condition, designed to reduce negative interpretations of ambiguity, target words always will be associated with the benign meanings of the cue-words, discouraging their negative interpretation. In the control condition, $50 \%$ of the target words will be associated with the benign meaning and $50 \%$ with the negative meanings of the cue-words.

A trained graduate research officer will supervise all CBM sessions, and participants will be randomly assigned to the active or control CBM groups. Previous studies (reviewed in the Introduction section) have shown that attentional and interpretative biases can be modified successfully using these procedures. In addition, there is evidence that exposure to daily CBM leads to extinction of negative bias within 2 weeks, ${ }^{35} 36$ thereby guiding the duration of our trial for efficacy while minimising treatment burden.

\section{Outcomes}

This trial has two primary outcomes of interest: depression and quality of life. We will use the CSDD to establish the presence of clinically significant symptoms of depression (CSDD $\geq 8$ ), measure changes in the severity of symptoms over 2 and 12 weeks, and to ascertain the remission of symptoms after 2 and 12 weeks (CSDD <8). The primary outcome of interest is the change, from baseline, in the severity of symptoms after 2 weeks of treatment. The CSDD is the most widely used instrument worldwide to assess depression in dementia. The 19-item scale is rated on a three-point score that ranges from absent to severe. The scale has robust psychometric properties, with good inter-rater reliability and internal consistency. $^{34}$ We are not aware of data describing minimal clinical important differences or minimal detectable changes for this scale. The CSDD is a rater-based assessment that takes about $10 \mathrm{~min}$ to complete. $^{34}$

The Quality of Life AD (QoL-AD) scale will be this study's measure of quality of life. ${ }^{37}$ It consists of 13 items that assess behavioural competence, psychological status, physical functioning and interpersonal environment that is of relevance to older adults. Each item offers four answers that range from poor (1) to excellent (4), with the total possible score ranging from 13 to 52 . Higher scores indicate better quality of life. The QoL-AD has the added advantage of offering patient and carer versions. The QoL-AD has robust psychometric properties. $^{37}$ Changes in QoL-AD scores from baseline represent another outcome of interest of this trial, and will be measured 2 (primary endpoint) and 12 weeks (secondary endpoint) after the baseline assessment.

Before the intervention starts, all participants will complete a baseline assessment of attentional and interpretative biases, using assessment variants of the procedures described for the intervention. In the attentional assessment, probes will appear $50 \%$ of the time in the position of neutral/happy faces and $50 \%$ of the time in the position of sad faces. Negative attentional bias will be indexed by degree of relative speeding to discriminate probes in the latter condition compared with the former. In the interpretative assessment, target words will be related to the benign meaning of the ambiguous cue word $50 \%$ of the time and to its negative meaning $50 \%$ of the time. Negative interpretative bias will be indexed by degree of relative speeding to identify target words in the latter condition compared to the former. This assessment will be repeated 2 and 12 weeks after the start of the intervention, so that change in attentional and interpretative biases can be ascertained (secondary outcomes).

We will also collect data on acceptability of the intervention, adherence to treatment and reasons for 
withdrawal from the study. We will measure acceptability by asking participants after 2 weeks: (1) please rate your experience with the CBM program: very unpleasant/ unpleasant/unsure/pleasant/very pleasant. (2) If your doctor recommends CBM treatment for you in the future, would you: refuse it/be unsure whether to do it/ be happy to do it again. Adherence will be measured automatically by the number of scheduled CBM session completed. We will consider that participants adhered to treatment if they completed at least 8 of 10 sessions. Partial and poor adherence will be defined by completion of 5-7 and less than 5 sessions, respectively. We will also ask participants who withdraw from the study to list the factors that might have contributed to their decision. Supportive collateral information will be sought from carers and treating physicians.

\section{Other study measures}

We will use a number of validated measures and procedures to collect demographic, lifestyle and clinical information from participants, including age, place of birth, time living in Australia, marital status, education, current and past occupations, living arrangements, religion and religion practices, weight and height, hobbies and financial concerns, smoking (never, past and current, amount and time), physical activity and alcohol consumption (standard units per day in a usual week), social support, prevalent medical conditions (diabetes, hypertension, dyslipidaemia, coronary heart disease, cerebrovascular disease, chronic respiratory diseases, arthritis and other rheumatic disorders, chronic pain, sensory impairment, cancer and others), as well as prescribed and over the counter medications (including antidepressants). The approach to data gathering will follow the same procedures that we have used successfully in other trials. ${ }^{38-40}$

The MMSE is the most widely used screening instrument for the assessment of cognitive function worldwide. ${ }^{33}$ Scores can range from 0 to 30 , with lower scores indicating increasing cognitive impairment. The MMSE requires about $10 \mathrm{~min}$ to complete, and will be carried out at screening and 12 weeks. Changes of MMSE scores over 2 and 12 weeks will be additional secondary outcomes of interest of this trial. Table 1 summarises the timeline for the collection of the outcomes of the study.

\section{Sample size}

Data from the placebo-controlled trial of mirtazapine and sertraline indicate that people with $\mathrm{DAD}$ treated with placebo improved, on average, six points $(\mathrm{SD}=4)$ on the CSDD after 13 weeks. ${ }^{15}$ We estimate that people treated with CBM should improve an additional three points (9 in total, $\mathrm{SD}=4$ ). A sample size of 76 people with $\mathrm{DAD}$ (38 per group) would give the study $90 \%$ power to declare this difference as significant ( $\alpha<5 \%$, two-tailed). We plan to recruit 80 participants with DAD (40 randomly assigned to each treatment group) and, pessimistically, anticipate that $15 \%$ of them will be lost during the study (our retention of people with $\mathrm{AD}$ in our trials is higher over a period of 6 months). ${ }^{41}$ In this case, the trial would be completed by 68 participants and would still have $87 \%$ power. We further anticipate that loss to follow-up will be minimal during the initial 2 weeks of treatment, although changes in scores may also be smaller at that point in time (three points for controls and six for intervention participants). If we lose $10 \%$ of our sample during the initial 2 weeks, primary outcome data would be available for 72 participants (36 per group) and the study would have $89 \%$ power to declare as significant such a difference between the groups. Furthermore, a sample of this size would give the study $84 \%$ power to declare as significant changes in QoL-AD scores of 1 point for controls and 4.5 points for active CBM participants over 3 months ( $\mathrm{SD}=5$ for both groups; positive changes indicate expected improvement). The changes in response bias (measured in milliseconds) are expected to be associated with a minimum effect size of 0.4 (Cohen's d), ${ }^{26}$ which would require about 50 trials per participant (within group comparison). As each CBM session includes 96 trials for attention (faces) and 96 trials for interpretative bias (words), the study will have ample power to investigate this outcome.

\section{Randomisation and allocation concealment}

Participants will be randomly assigned to control and active CBM according to a list of random numbers generated by computer in blocks of 10 . The allocation ratio

Table 1 Timeline for the collection of outcomes for RAPID

\begin{tabular}{lllll}
\hline & Screening & Baseline & Week 2 & Week 12 \\
\hline Sociodemographic data & Yes & - & - & - \\
Lifestyle & Yes & - & - & - \\
Medical conditions and medications & Yes & - & - & - \\
Daily alcohol consumption & Yes & - & - & - \\
Confirming diagnosis of AD & Yes & - & - & - \\
Mini-Mental State Examination & Yes & - & Yes & Yes \\
Cornell Scale for Depression in Dementia & Yes & Yes & Yes & Yes \\
Quality of Life AD & - & Yes & Yes & Yes \\
Attentional and interpretative biases & - & Yes & Yes & Yes \\
\hline AD, Alzheimer's disease. & & &
\end{tabular}


will be 1:1. An independent biostatistician working at the Western Australian Centre for Health and Ageing will generate the random allocation sequence. The sequence code will then be used to automatically select the appropriate intervention for the participant (control or active CBM) from our server (ie, the id number will be linked to the randomisation code and the relevant intervention will be activated when the user id is entered on the computer). Neither the participant nor research staff will be aware of the independently controlled randomisation code. Participants will be advised about the study's aims and procedures (which will be exactly the same for all participants), but not about the details characterising the control and the active intervention. In addition, the staff member supervising the CBM sessions will not be involved in the collection of outcome data. This professional will be directed not to discuss any aspects of the intervention with other research staff. Previous trials have shown this approach is sufficient to ensure masking. ${ }^{42}$ Group assignment codes will only be opened after the last end point of interest of the last participant in the trial is collected.

\section{Data collection and management}

Attentional and interpretative bias data will be collected automatically by the study computer during the assessment. In order to optimise the validity of the data collected, participants will be offered three breaks during each assessment once 25\%, 50\% and $75 \%$ of each session is completed. They may elect to take or override such offers of rest by asking the research officer to press the space bar, which once pressed will re-start the task. If response to a trial (faces or words) takes longer than $5 \mathrm{~s}$, that trial will be disregarded in the calculation of attentional and interpretative bias (long response latencies indicate distraction rather than bias).

The collection of end points will occur regardless the intervention adherence of participants. If participants become unable, or unwilling, to present to the research site for assessment, a home assessment will be offered. OPA will train research staff in the use of the CSDD and QoL-ADagreement of 0.8 or greater on weighted $\kappa$ will be required before the research officer is allowed to collect end points independently (minimum of five assessments).

\section{Statistical methods}

We will use means and SDs to describe continuous variables with normal distribution, medians and IQRs for ordinal variables, and frequency tables for categorical variables. We will use t tests to compare the change in CSDD and QoL-AD scores between baseline and week 2. If statistical adjustments are required because of imbalance in other study measures (eg, gender distribution), we will use analysis of co-variance. We will use multilevel mixed models to analyse changes in CSDD, QoL and attentional and interpretative biases from baseline to weeks 2 and 12 . We will investigate the interaction between group and time effects (statistical adjustments will be made, if necessary).
This analysis is intention to treat. Multiple imputations (imputed chain equations) will be employed for the analysis of week 2 data, if necessary.

\section{Data monitoring}

No interim analyses are planned for this trial.

\section{Harms}

We do not anticipate that the procedures associated with the intervention have potential to causing clinically significant harm. Participants will be offered the opportunity of having breaks in order to minimise fatigue, although each intervention session is expected to last no more than $10 \mathrm{~min}$. There will also be an opportunity for coffee and toilet breaks between intervention sessions and throughout the assessment procedures.

\section{Auditing}

Once a participant is randomised, the study computer will set up the study agenda for that person automaticallyadherence will be recorded, analysed and reported. A quarterly internal audit will evaluate possible violations of protocol and research schedule, as well as integrity of the study's database (including accuracy of the data recorded). Electronic records will be backed up and maintained in a secure server at the University of Western Australia. Paper records of assessments will be kept for a minimum period of 5 years following the collection of the last end point of the trial. No external auditing is planned.

\section{DISCUSSION}

$\mathrm{AD}$ is a leading cause of disability worldwide and the course of the illness is often complicated by the presence of depressive symptoms. Currently available antidepressant medications are not effective to treating DAD, so that efficacious alternatives are needed. There is strong empirical support for testing the efficacy of CBM in this population, and the design of our study has followed SPIRIT and CONSORT guidelines in order to ensure that the trial generates high quality data that address its objectives appropriately. ${ }^{43} 44$

We have designed a CBM intervention that does not require the use of a keyboard or mouse (ie, response entails the pressing of one of two buttons of the response box) and imposes only minimal demands on the cognitive function of participants. We expect that this will increase the acceptability of and adherence to the intervention in this group of older participants.

\section{Study limitations}

The definition of depression in this study will be guided by the use of a validated cut-point on the CSDD rather than DSM-IV, DSM-5 or ICD-10 criteria for the diagnosis of a depressive episode. ${ }^{34}$ We chose this approach for two reasons: (1) the DSM and ICD criteria for the diagnosis of a major depressive episode associated with $\mathrm{AD}$ have uncertain validity, even though provisional 
alternate criteria have been proposed ${ }^{45}$;6 (2) pragmatic trials for the treatment of DAD have used CSDD scores of 8 or more to define clinically significant depression, with the use of the scale offering the additional advantage of measuring change in the severity of symptoms over time ${ }^{15}$ and of allowing direct comparisons between the results of previous studies and ours.

This trial will be limited to people with DAD of mild to moderate severity. Consequently, its findings will not be generalisable to older adults with severe AD. This criterion for inclusion took into account the ability of participants to offer informed consent, and the relative integrity of brain systems involved in implicit learning during the early stages of illness. CBM works through implicit learning, which might be undermined late in the course of $\mathrm{AD}{ }^{47}$

The duration of the intervention in RAPID will be limited to 2 weeks, which is the time required to modify cognitive biases in adults free of cognitive impairment. $^{35} 36$ We anticipate that a similar timeline will be required to treat older adults with $\mathrm{DAD}$, but cannot be certain, at this stage, that this will be the case. In addition, at this point in time, there is no direct evidence that the CBM paradigm will work as well in people with $\mathrm{AD}$ as it does in younger adults free of cognitive impairment. Our trial will yield such evidence, and this will allow us to ascertain if the effect (or lack of effect) of the intervention was due to the expected shifting of attentional and interpretative biases. We will monitor attentional and interpretative biases throughout the study, and this will enable us to measure the association between extinction of bias and decline in the severity of depressive symptoms. Moreover, we have included a 12-week follow-up assessment to investigate the medium term sustainability of the changes in bias and mood of participants.

Finally, we acknowledge that our power calculations are based on an expectation of clinically significant improvement rather than existing preliminary data. Our predictions assume that the intervention will be associated with a relative reduction of an additional 3 out of a possible 38 points compared with controls. Consequently, this trial will be underpowered to declare as statistically significant smaller differences between the groups.

\section{Author affiliations}

${ }^{1}$ Western Australian Centre for Heath \& Ageing (M573), Centre for Medical Research of the Perkins Institute for Medical Research, University of Western Australia, Perth, Western Australia, Australia

${ }^{2}$ School of Psychiatry \& Clinical Neurosciences, University of Western Australia, Perth, Western Australia, Australia

${ }^{3}$ Department of Psychiatry, Royal Perth Hospital, Perth, Western Australia, Australia

${ }^{4}$ School of Psychology, University of Western Australia, Perth, Western Australia, Australia

${ }^{5}$ School of Medicine and Pharmacology, University of Western Australia, Perth, Western Australia, Australia

${ }^{6}$ Department of Geriatric Medicine, Royal Perth Hospital, Perth, Western Australia, Australia
Contributors OPA and CM conceived and designed the study. LF, AF, BG and CE-B contributed to the development of the protocol. OPA, LF, AF and CE-B obtained the start-up funding for the trial. $B G$ retrieved and organised the material for the CBM tasks. OPA drafted the manuscript, which all authors reviewed critically and approved for submission.

Funding RAPID is currently funded by an unrestricted start-up grant from the Theodore and Isabella Wearne Charitable Trust.

Competing interests None.

Ethics approval The Human Research Ethics Committee of the Royal Perth Hospital approved the research protocol and procedures of the study (protocol number 14-036, 26 March 2014), which follow the principles of the Declaration of Helsinki.

Provenance and peer review Not commissioned; externally peer reviewed.

Open Access This is an Open Access article distributed in accordance with the Creative Commons Attribution Non Commercial (CC BY-NC 4.0) license, which permits others to distribute, remix, adapt, build upon this work noncommercially, and license their derivative works on different terms, provided the original work is properly cited and the use is non-commercial. See: http:// creativecommons.org/licenses/by-nc/4.0/

\section{REFERENCES}

1. Ferri $\mathrm{CP}$, Prince $\mathrm{M}$, Brayne $\mathrm{C}$, et al. Global prevalence of dementia: a Delphi consensus study. Lancet 2005;366:2112-17.

2. Vos $T$, Flaxman AD, Naghavi M, et al. Years lived with disability (YLDs) for 1160 sequelae of 289 diseases and injuries 1990-2010: a systematic analysis for the Global Burden of Disease Study 2010. Lancet 2012;380:2163-96.

3. Lloyd-Sherlock P. Population ageing in developed and developing regions: implications for health policy. Soc Sci Med 2000;51:887-95.

4. Bartlett H, Gray L, Byrne G, et al. Dementia Research Mapping Project: final report. Canberra: Department of Health and Ageing, 2006.

5. Burns A, Jacoby R, Levy R. Psychiatric phenomena in Alzheimer's disease. Br J Psychiatry 1990;157:72-94.

6. Starkstein SE, Dragovic M, Jorge R, et al. Diagnostic criteria for depression in Alzheimer disease: a study of symptom patterns using latent class analysis. Am J Geriatr Psychiatry 2011;19:551-8.

7. Spalletta G, Musicco M, Padovani A, et al. Neuropsychiatric symptoms and syndromes in a large cohort of newly diagnosed, untreated patients with Alzheimer disease. Am J Geriatr Psychiatry 2010;18:1026-35.

8. Black W, Almeida OP. A systematic review of the association between the behavioral and psychological symptoms of dementia and burden of care. Int Psychogeriatr 2004;16:295-315.

9. Bosboom PR, Alfonso H, Almeida OP. Determining the predictors of change in quality of life for people with Alzheimer disease. Alzheimer Dis Assoc Disord 2013;27:363-71.

10. Rapp MA, Schnaider-Beeri M, Wysocki M, et al. Cognitive decline in patients with dementia as a function of depression. Am J Geriatr Psychiatry 2011;19:357-63.

11. Ito $\mathrm{K}$, Ahadieh $\mathrm{S}$, Corrigan $\mathrm{B}$, et al. Disease progression meta-analysis model in Alzheimer's disease. Alzheimers Dement 2010;6:39-53.

12. Birks J. Cholinesterase inhibitors for Alzheimer"s disease. Cochrane Database Syst Rev 2006;(1):CD005593.

13. Rodda J, Morgan S, Walker Z. Are cholinesterase inhibitors effective in the management of the behavioral and psychological symptoms of dementia in Alzheimer's disease? a systematic review of randomized, placebo-controlled trials of donepezil, rivastigmine and galantamine. Int Psychogeriatr 2009;21:813-24.

14. Zarate CA Jr, Singh JB, Quiroz JA, et al. A double-blind, placebo-controlled study of memantine in the treatment of major depression. Am J Psychiatry 2006;163:153-5.

15. Banerjee S, Hellier J, Dewey M, et al. Sertraline or mirtazapine for depression in dementia (HTA-SADD): a randomised, double-blind, placebo-controlled trial. Lancet 2011;378:403-11.

16. Nelson JC, Devanand DP. A systematic review and meta-analysis of placebo-controlled antidepressant studies in people with depression and dementia. J Am Geriatr Soc 2011;59:577-85.

17. Butler AC, Chapman JE, Forman EM, et al. The empirical status of cognitive-behavioral therapy: a review of meta-analyses. Clin Psychol Rev 2006;26:17-31. 
18. Sudak DM. Cognitive behavioral therapy for depression. Psychiatr Clin North Am 2012;35:99-110.

19. Vittengl JR, Clark LA, Dunn TW, et al. Reducing relapse and recurrence in unipolar depression: a comparative meta-analysis of cognitive-behavioral therapy's effects. J Consult Clin Psychol 2007;75:475-88.

20. Hollon SD, Evans MD, Dereubeis RJ. Cognitive mediation of relapse prevention following treatment for depression: implications of differential risk. In: Ingram RE, ed. Psychological aspects of depression. New York: Plenum, 1990:117-36.

21. Macleod C, Holmes EA. Cognitive bias modification: an intervention approach worth attending to. Am J Psychiatry 2012;169:118-20.

22. Macleod C. Cognitive bias modification procedures in the management of mental disorders. Curr Opin Psychiatry 2012;25:114-20.

23. Gotlib IH, Krasnoperova E, Yue DN, et al. Attentional biases for negative interpersonal stimuli in clinical depression. J Abnorm Psychol 2004;113:121-35.

24. Grey SJ, Mathews AM. Cognitive bias modification-priming with an ambiguous homograph is necessary to detect an interpretation training effect. J Behav Ther Exp Psychiatry 2009;40:338-43.

25. MacLeod C, Rutherford E, Campbell L, et al. Selective attention and emotional vulnerability: assessing the causal basis of their association through the experimental manipulation of attentional bias. J Abnorm Psychol 2002;111:107-23.

26. See J, MacLeod C, Bridle R. The reduction of anxiety vulnerability through the modification of attentional bias: a real-world study using a home-based cognitive bias modification procedure. J Abnorm Psychol 2009;118:65-75

27. Hirsch CR, MacLeod C, Mathews A, et al. The contribution of attentional bias to worry: distinguishing the roles of selective engagement and disengagement. J Anxiety Disord 2011;25:272-7.

28. Najmi S, Amir N. The effect of attention training on a behavioral test of contamination fears in individuals with subclinical obsessivecompulsive symptoms. J Abnorm Psychol 2010;119:136-42.

29. Wells TT, Beevers CG. Biased attention and dysphoria: manipulating selective attention reduces subsequent depressive symptoms. Cogn Emot 2010;24:719-28.

30. Williams AD, Blackwell SE, Mackenzie A, et al. Combining imagination and reason in the treatment of depression: RCT of internet CBM and CBT. J Consult Clin Psychol 2013;81:793-9.

31. Ballesteros S, Reales JM. Intact haptic priming in normal aging and Alzheimer's disease: evidence for dissociable memory systems. Neuropsychologia 2004:42:1063-70.

32. McKhann G, Drachman D, Folstein M, et al. Clinical diagnosis of Alzheimer's disease: report of the NINCDS-ADRDA Work Group under the auspices of Department of Health and Human Services Task Force on Alzheimer's Disease. Neurology 1984;34:939-44.

33. Folstein MF, Folstein SE, McHugh PR. "Mini-mental state". A practical method for grading the cognitive state of patients for the clinician. J Psychiatr Res 1975;12:189-98.

34. Alexopoulos GS, Abrams RC, Young RC, et al. Cornell Scale for depression in dementia. Biol Psychiatry 1988;23:271-84.

35. Blackwell SE, Holmes EA. Modifying interpretation and imagination in clinical depression: case series using cognitive bias modficiation. Appl Cogn Psychol 2010;24:338-50.

36. Browning M, Holmes EA, Charles M, et al. Using attentional bias modification as a cognitive vaccine against depression. Bio Psychiatry 2012;72:572-9.

37. Logsdon RG, Gibbons LE, McCurry SM, et al. Assessing quality of life in older adults with cognitive impairment. Psychosom Med 2002:64:510-19.

38. Norman PE, Flicker L, Almeida OP, et al. Cohort Profile: The Health In Men Study (HIMS). Int J Epidemiol 2009;38:48-52.

39. Ford AH, Flicker L, McCaul K, et al. The B-VITAGE trial: a randomized trial of homocysteine lowering treatment of depression in later life. Trials 2010;11:8.

40. Almeida OP, Pirkis J, Kerse N, et al. A randomized trial to reduce the prevalence of depression and self-harm behavior in older primary care patients. Ann Fam Med 2012;10:347-56.

41. Cyarto EV, Cox KL, Almeida OP, et al. FABS II: protocol for a RCT evaluating the effect of physical activity on cognitive function in patients with Alzheimer's disease. Trials 2010;11:120.

42. Amir N, Beard C, Taylor CT, et al. Attention training in individuals with generalized social phobia: a randomized controlled trial. $J$ Consult Clin Psychol 2009;77:961-73.

43. Schulz KF, Altman DG, Moher D, et al. CONSORT 2010 statement: updated guidelines for reporting parallel group randomised trials. BMJ 2010;340:c332.

44. Chan AW, Tetzlaff JM, Altman DG, et al. SPIRIT 2013: new guidance for content of clinical trial protocols. Lancet 2013;381:91-2.

45. Olin JT, Katz IR, Meyers BS, et al. Provisional diagnostic criteria for depression of Alzheimer disease: rationale and background. $A m ~ J$ Geriatr Psychiatry 2002;10:129-41.

46. Olin JT, Schneider LS, Katz IR, et al. Provisional diagnostic criteria for depression of Alzheimer disease. Am J Geriatr Psychiatry 2002;10:125-8

47. Fleischman DA, Gabrieli JD. Repetition priming in normal aging and Alzheimer's disease: a review of findings and theories. Psychol Aging 1998;13:88-119. 